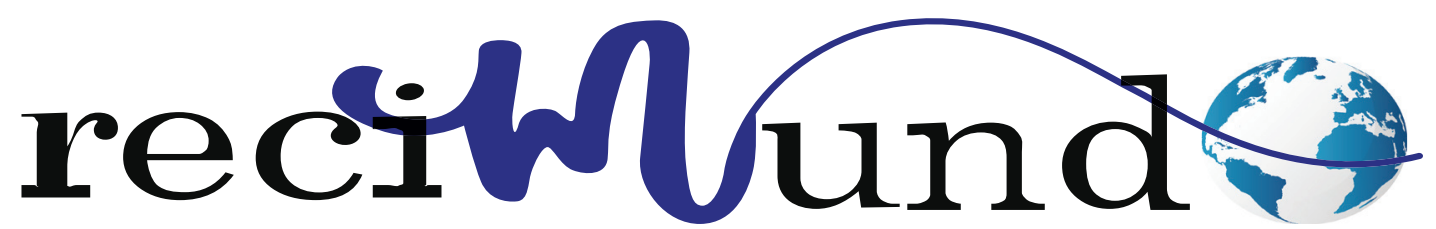

Revista Científica Mundo de la Investigación y el Conocimiento

DOl: 10.26820/recimundo/5.(2).julio.2021.188-196

URL: https://recimundo.com/index.php/es/article/view/1243

EDITORIAL: Saberes del Conocimiento

REVISTA: RECIMUNDO

ISSN: 2588-073X

TIPO DE INVESTIGACIÓN: Artículo de revisión

CóDIGO UNESCO: 32 Ciencias Médicas

PAGINAS: 188-196

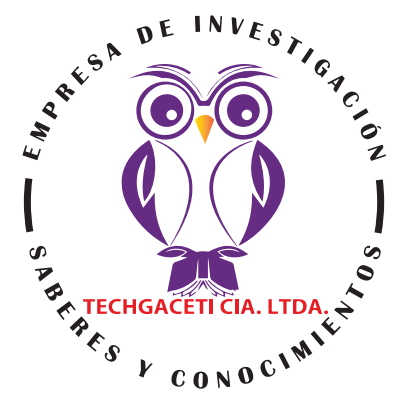

\title{
Causas de absceso del musculo psoas
}

Causes of psoas muscle abscess

Causas de abscesso do músculo psoas

Alex Armando Ronquillo Saavedra'; Daniela Natalie Rodríguez Cabrera²; Carlos Bruno Guerrero Cedeño ${ }^{3}$

RECIBIDO: 11/04/2021 ACEPTADO: 15/06/2021 PUBLICADO: 30/07/2021

1. Médico de la Universidad de Guayaquil; Investigador Independiente; Guayaquil, Ecuador; aros_92@hotmail.com (iD https://orcid.org/0000-0001-8046-6780

2. Médico de la Universidad de Guayaquil; Investigador Independiente; Guayaquil, Ecuador; dani_rodriguez91@hotmail.es (iD) https://orcid.org/0000-0003-2804-8738

3. Médico de la Universidad de Guayaquil; Investigador Independiente; Guayaquil, Ecuador; drcarlosguerrero@hotmail.es (iD) https://orcid.org/0000-0002-7664-6989

CORRESPONDENCIA

Alex Armando Ronquillo Saavedra

aros_92@hotmail.com

Guayaquil, Ecuador

(c) RECIMUNDO; Editorial Saberes del Conocimiento, 2021 


\section{RESUMEN}

Los abscesos del músculo psoas representan una patología con muy baja incidencia a nivel mundial, por lo que se requiere de mucho diagnóstico para su abordaje y generalmente se relaciona con enfermedades gastrointestinales. El diagnóstico es tardío debido a que sus manifestaciones clínicas son poco específicas, lo que trae como consecuencia, un pronóstico particularmente malo en la mayoría de los casos. Se presenta como una acumulación de pus que se ubica en el musculo psoas iliaco y es producida por la continuidad desde estructuras adyacentes o por diseminación hematógena, sin embargo, se considera que su etiología es diversa. En tal sentido, es sumamente importante tomar en consideración la posibilidad de que sea de origen abdominal la presencia de esta patología, debido a que el drenaje quirúrgico urgente y agresivo es el único tratamiento eficaz en estas situaciones. De acuerdo con la baja incidencia o poca frecuencia de la aparición de este tipo de abscesos, se tiene como objetivo, evaluar sus causas a través, de una revisión bibliográfica donde se puntualice el tema y se presenten una serie de casos disponibles en la literatura que dan cuerpo y brindan información importante al respecto.

Palabras clave: Absceso, musculo, psoas, psoas iliaco.

\section{ABSTRACT}

Abscesses of the psoas muscle represent a pathology with a very low incidence worldwide, which is why it requires a lot of diagnosis for its approach and is generally related to gastrointestinal diseases. Diagnosis is late because its clinical manifestations are not very specific, which results in a particularly poor prognosis in most cases. It presents as an accumulation of pus that is located in the iliac psoas muscle and is produced by continuity from adjacent structures or by hematogenous dissemination, however, its etiology is considered to be diverse. In this sense, it is extremely important to take into consideration the possibility that the presence of this pathology is of abdominal origin, since urgent and aggressive surgical drainage is the only effective treatment in these situations. For this reason and according to the low incidence or infrequency of the appearance of this type of abscess, the objective is to evaluate its causes through a bibliographic review where the subject is specified and a series of available cases are presented in the literature that give substance and provide important information in this regard.

Keywords: Abscess, muscle, psoas, iliac psoas.

\section{RESUMO}

Os abscessos do músculo psoas representam uma patologia de baixíssima incidência mundial, por isso requerem muitos diagnósticos para sua abordagem e geralmente estão relacionados a doenças gastrointestinais. O diagnóstico é tardio porque suas manifestações clínicas são pouco específicas, o que resulta em um prognóstico particularmente ruim na maioria dos casos. Apresenta-se como um acúmulo de pus que se localiza no músculo psoas ilíaco e é produzido pela continuidade de estruturas adjacentes ou por disseminação hematogênica, porém sua etiologia é considerada diversa. Nesse sentido, é de extrema importância levar em consideração a possibilidade de a presença dessa patologia ser de origem abdominal, uma vez que a drenagem cirúrgica urgente e agressiva é o único tratamento eficaz nessas situações. Por esse motivo e de acordo com a baixa incidência ou infrequência do aparecimento deste tipo de abscesso, o objetivo é avaliar suas causas por meio de uma revisão bibliográfica onde o assunto é especificado e uma série de casos disponíveis são apresentados na literatura que dão substância e fornecer informações importantes a esse respeito.

Palavras-chave: Abscesso, músculo, psoas, psoas ilíaco. 


\section{Introducción}

El músculo psoas y el músculo ilíaco se consideran como un solo músculo llamado psoas ilíaco, "ubicado en un espacio extraperitoneal llamado compartimento iliopsoas. El músculo psoas es largo y fusiforme, ubicado lateralmente en la columna lumbar, en ambos lados" (Mallick, Thoufeeq, \& Rajendran, 2004).

Pasa por debajo del ligamento inguinal y anterior a la articulación coxo-femoral. Termina con inserción tendinosa en el trocánter pequeño de la extremidad femural proximal. Este músculo está inervado por las ramas de los nervios espinales L2-L4 y es el flexor más importante del muslo (Mallick, Thoufeeq, \& Rajendran, 2004).

Se considera que debido a su localización el absceso del músculo psoas es una complicación común de la tuberculosis vertebral. "Adicionalmente, el músculo psoas está adyacente a muchos órganos como: el colon sigmoide, el apéndice, el yeyuno, el uréter, la aorta abdominal, el riñón, el páncreas, la columna y los ganglios linfáticos" (Shieldsa, Robinsonb, \& Crowley, 2012). Por lo tanto, un proceso infeccioso de cualquiera de estos órganos podría desencadenar en el músculo psoas y la infección a este nivel puede ocurrir por diseminación hematógena desde un lugar anatómicamente de origen lejano.

Estos abscesos se localizan retrofascialmente, más que retroperitoneal, "ya que el músculo psoas es posterior al músculo psoas fascia transversal, que representa el borde posterior del retroperitoneo" (Shieldsa, Robinsonb, \& Crowley, 2012). La incidencia de esta patología es baja, pero ha aumentado en los últimos años, con el uso generalizado de la tomografía computarizada en el diagnóstico de diversas enfermedades.
Puede presentarse con una sintomatología variada en cualquier especialidad aguda, debido a la relativa rareza y a las características inespecíficas, lo que ocasiona retrasos en el diagnóstico y el tratamiento eficaz de la enfermedad. En tal sentido, durante el desarrollo del proceso investigativo se busca reunir información que brinde una revisión concisa y sistemática de la etiología, manejo y las causas relacionadas al absceso psoas.

\section{Metodología}

Para el desarrollo de este proceso investigativo, se plantea como metodología la encaminada hacia una orientación científica particular que se encuentra determinada por la necesidad de indagar en forma precisa y coherente una situación, en tal sentido Davila, (2015) define la metodología "como aquellos pasos anteriores que son seleccionados por el investigador para lograr resultados favorables que le ayuden a plantear nuevas ideas" (p.66)

Lo citado por el autor, lleva a entender que el desarrollo de la acción investigativa busca simplemente coordinar acciones enmarcadas en una revisión bibliográfica con el fin de complementar ideas previas relacionadas Causas de absceso del musculo a través de una revisión de literatura, para así finalmente elaborar un cuerpo de consideraciones generales que ayuden a ampliar el interés propuesto.

\section{Tipo de Investigación}

Dentro de toda práctica investigativa, se precisan acciones de carácter metodológico mediante las cuales se logra conocer y proyectar los eventos posibles que la determinan. En este sentido, la presente investigación corresponde al tipo documental, definido por Castro (2016), "se ocupa del estudio de problemas planteados a nivel teórico, la información requerida para abordarlos se encuentra básicamente en mate- 
riales impresos, audiovisuales y / o electrónicos". (p.41).

En consideración a esta definición, la orientación metodológica incluye la oportunidad de cumplir con una serie de actividades inherentes a la revisión y lectura de diversos documentos, donde se encuentran ideas explicitas relacionadas con los tópicos encargados de identificar una característica inmersa en el estudio. Por lo tanto, se realizaron continuas interpretaciones con el claro propósito de revisar aquellas apreciaciones propuestas por diferentes investigadores en relación al tema de interés, para luego dar la respectiva argumentación a los planteamientos, en función a las necesidades encontradas en la investigación, apoyados en las herramientas tecnológicas para la búsqueda de trabajos con valor científico disponibles en la web que tenían conexión con el objetivo principal de la investigación.

\section{Fuentes Documentales}

El análisis correspondiente a las características que predomina en el tema seleccionado, llevan a incluir diferentes fuentes documentales encargadas de darle el respectivo valor científico y en ese sentido cumplir con la valoración de los hechos a fin de generar nuevos criterios que sirven de referencia a otros procesos investigativos. Para Castro,(2016) las fuentes documentales incorporadas en la investigación documental o bibliográfica, "representa la suma de materiales sistemáticos que son revisados en forma rigurosa y profunda para llegar a un análisis del fenómeno" (p.41). Por lo tanto, se procedió a cumplir con la lectura previa determinada para encontrar aquellos aspectos estrechamente vinculados con el tema, con el fin de explicar mediante un desarrollo las respectivas apreciaciones generales de importancia.

\section{Técnicas para la Recolección de la Infor- mación}

La conducción de la investigación para ser realizada en función a las particularidades que determinan a los estudios documentales, tiene como fin el desarrollo de un conjunto de acciones encargadas de llevar a la selección de técnicas estrechamente vinculadas con las características del estudio. Bolívar, (2015), refiere, que es "una técnica particular para aportar ayuda a los procedimientos de selección de las ideas primarias y secundarias". (p.71).

Tal como lo expresa, Bolívar, (2015) "Las técnicas documentales proporcionan las herramientas esenciales y determinantes para responder a los objetivos formulados y llegar a resultados efectivos" (p. 58). Es decir, para responder con eficiencia a las necesidades investigativas, se introdujeron como técnica de recolección el método inductivo, que hizo posible llevar a cabo una valoración de los hechos de forma particular para llegar a la explicación desde una visión general. El autor Bolívar, (2015) tambien expresa que las técnicas de procesamiento de datos en los estudios documentales "son las encargadas de ofrecer al investigador la visión o pasos que deben cumplir durante su ejercicio, cada una de ellas debe estar en correspondencia con el nivel a emplear" (p. 123). Esto indica, que para llevar a cabo el procesamiento de los datos obtenidos una vez aplicadas las técnicas seleccionadas, tales como: fichas de resumen, textual, registros descriptivos entre otros, los mismos se deben ajustar al nivel que ha sido seleccionado.

\section{Resultado}

\section{Manifestaciones clínicas}

Actualmente se describen dos tipos de abscesos del músculo psoas: primario y secundario. "Los abscesos primarios aparecen debido a la diseminación linfática o hema-

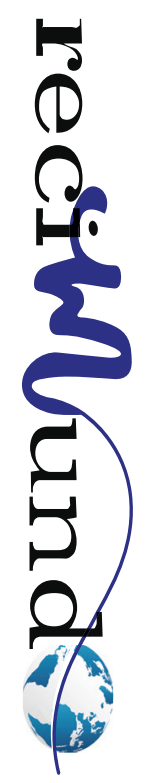


tógena de un microorganismo de una infección localizada remota. Los secundarios ocurren debido a la propagación directa de un proceso infeccioso vecinal" (Shieldsa, Robinsonb, \& Crowley, 2012)

Los abscesos primarios del músculo psoas ocurren con mayor frecuencia en niños donde generalmente se confunden con artritis séptica de la articulación de la cadera, "lo que representa aproximadamente el 30\% de todos los casos, o en pacientes con otras patologías asociadas como diabetes, consumidores crónicos de alcohol o drogas intravenosas, insuficiencia renal, antecedentes de malignidad o desnutrición" (Mallick, Thoufeeq, \& Rajendran, 2004). "Según estudios se describe la propagación de una infección a partir de una infección remota (hematógena) o un hematoma formado en el músculo que se ha infectado" (Thongngarm \& Mcmurray, 2001 ).

Los autores Mallick, Thoufeeq, \& Rajendran, 2004 exponen, "Ios abscesos secundarios del músculo psoas ocurren debido a la propagación casi cercana de una infección localizada desde un órgano cercano". Entre las que se mencionan:

- linfadenitis infecciosa local.

- Sacroileitis infecciosa, osteomielitis, artritis séptica.

- Aneurisma femoral y abdominal infectado, cateterismo de los vasos locales importantes.

- Infecciones del tracto urinario, tumores cancerosos / no cancerosos infectados, litotricia de choque extracorpóreo, dispositivos intrauterinos.

- Patología colorrectal: cáncer, enfermedad de Crohn, diverticulitis, apendicitis

Se requiere un examen clínico completo y correcto para diagnosticar rápidamente esta condición. Si el paciente mantiene una posición antálgica (el paciente está acostado boca arriba, con el muslo ligeramente flexionado y girado hacia afuera), el diag- nóstico es casi obvio. Esta posición reduce la presión en el compartimento del psoas ilíaco y reduce el nivel del dolor (Shieldsa, Robinsonb, \& Crowley, 2012).

El cuadro clínico que presentan estos pacientes suele ser variable e inespecífico. "La tríada diagnóstica clásica: fiebre, dolor lumbar y cojera está presente en aproximadamente el $30 \%$ de los pacientes" (Ion, Socea, Bolocani, Paduraru, \& Andronic, 2020). Otros síntomas pueden ser dolor abdominal difuso, náuseas y pérdida de peso. Debido a su ubicación e inervación, el dolor puede irradiarse a caderas y muslos. "El cuadro clínico también puede incluir una formación pseudotumoral inmediatamente debajo del ligamento inguinal, que representa un elemento de diagnóstico diferencial con la hernia femoral" (Mallick, Thoufeeq, \& Rajendran, 2004)

También hay signos claros que marcan el daño en el músculo psoas: el signo del psoas. "Se describe como un aumento de la intensidad del dolor abdominal en la flexión pasiva del muslo con el paciente en decúbito dorsal. Este signo tiene una sensibilidad del $24 \%$ en el diagnóstico de abscesos del psoas" (Demuren \& Abomelha, 2002).

\section{Microbiología}

Staphylococcus aureus "es el microorganismo más comúnmente identificado en los abscesos del psoas y causa aproximadamente el $88 \%$ de los abscesos primarios" (Abada, Solanoa, \& Ibarmiaa, 2000).

Los abscesos secundarios del músculo psoas suelen estar determinados por gérmenes que pertenecen a Streptococcus $(4,9 \%)$ o E. coli $(2,8 \%)$. Mycobacterium tuberculosis es el microorganismo notificado con más frecuencia en los abscesos del psoas que se presentan en pacientes de países en desarrollo (Abada, Solanoa, \& Ibarmiaa, 2000). 
En tal sentido, se puede decir que el patógeno que provoca la infección del órgano primario es el mismo que provoca el absceso del músculo psoas en el caso de abscesos secundarios.

\section{Laboratorio e imagenología}

Antes de administrar cualquier tratamiento, el paciente también puede presentar un recuento elevado de glóbulos blancos, anemia y niveles elevados de proteína $C$ reactiva acompañados de niveles elevados de VSG. Los hemocultivos también pueden ser positivos para el mismo germen que se ha identificado en el absceso.

Las investigaciones radiológicas iniciales pueden tener como objetivo diagnosticar otras patologías: urografía intravenosa, enema de bario. Desafortunadamente, estos no son específicos de los abscesos del psoas y no ayudan en el proceso de diagnóstico. En ocasiones, en la infección por microorganismos anaerobios, pueden aparecer burbujas de gas en el retroperitoneo, lo que puede establecer el diagnóstico (Mallick, Thoufeeq, \& Rajendran, 2004).

Sin embargo, una investigación de primera mano puede ser la ecografía. Es fácil de lograr y no es invasivo para el paciente. "La desventaja de este método es que solo visualiza los abscesos del psoas en aproximadamente el $60 \%$ de los casos. El espacio retroperitoneal puede ser difícil de visualizar debido al gas intestinal" (Van Den Berge \& Kuipers, 2005). El estándar de oro en el diagnóstico por imágenes de los abscesos del músculo psoas es la tomografía computarizada.

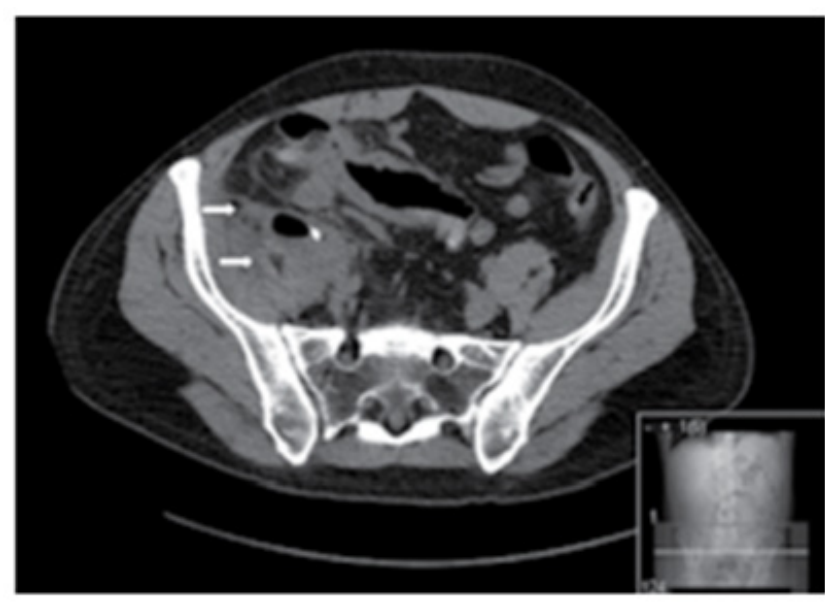

Figura 1. Tomografía computarizada para el diagnóstico de absceso de psoas derecho.

Fuente: (Ruiz, Candel, Ruiz, \& Peña, 2014).

Hay autores que afirman que "la resonancia magnética nuclear tendría resultados mucho mejores en el diagnóstico de esta patología, ya que pueden visualizar la pared del absceso sin necesidad de contraste intravenoso" (Mallick, Thoufeeq, \& Rajendran, 2004). Aunque actualmente hay una multitud de métodos de diagnóstico por imagen para los abscesos del músculo psoas, todavía existe la posibilidad de un diagnóstico falso negativo, por lo que los médicos pueden ignorar esta patología.

\section{Tratamiento}

El tratamiento de los abscesos del músculo psoas sigue siendo un problema médico y quirúrgico. Según Wong \& Lam, (2013), "existen varios métodos de tratamiento para los abscesos del músculo psoas: antibióticos de amplio espectro, drenaje del absceso por TC o punción guiada por ecografía, intervención quirúrgica y colocación de un tubo de drenaje en la cavidad del absceso". Generalmente, estos métodos se entrelazan debido a que se tiene que administrar antibióticos dirigidos, "es obligatorio obtener un cultivo del absceso y también conocer su sensibilidad a los antibióticos, por lo que está indicado el drenaje percutáneo guiado

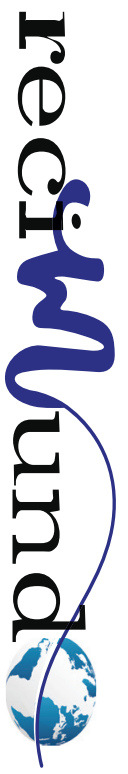


por tomografía computarizada o la cirugía para obtener material biológico" (Wong \& Lam, 2013). En los abscesos primarios, la mayoría de las veces también hay bacteriemia, por lo que los hemocultivos serán positivos para el mismo microorganismo.

En este momento, hay dos tipos de tratamiento, que con mayor frecuencia se usan juntos: el curso apropiado de antibióticos y el drenaje del absceso.

La elección inicial de antibióticos suele ser "ciega". Por lo general, se administran antibióticos de amplio espectro como primera línea de tratamiento hasta que se tiene la confirmación del tipo y la sensibilidad a los antibióticos del microorganismo encontrado en hemocultivos o cultivos de líquido de abscesos (Van Den Berge \& Kuipers, 2005).

Una vez que se tiene la confirmación del microorganismo que causa el absceso, el tratamiento se cambia a los antibióticos que son más efectivos en el tipo específico de microorganismo. "El tratamiento es diferente para los abscesos del músculo psoas ilíaco primario o secundario. En los abscesos primarios, deben administrarse antibióticos antiestafilocócicos antes de los resultados del cultivo (Van Den Berge \& Kuipers, 2005). "En los abscesos secundarios, deben administrarse antibióticos de amplio espectro antes de los resultados del cultivo (p. Ej., Penicilina antiestafilocócica, aminoglucósidos, clindamicina)" (Wong \& Lam, 2013).

De acuerdo con Mallick, Thoufeeq, \& Rajendran, (2004), "existen 2 métodos de drenaje para los abscesos del músculo psoas ilíaco". Estos son:

1. El drenaje percutáneo guiado por tomografía computarizada: el cual "es un procedimiento menos invasivo para el paciente, con una baja tasa de morbilidad y mortalidad y está clasificado como el método de elección. para drenar este absceso" (Wong \& Lam, 2013). Se aso- cia con antibióticos, así como también se puede utilizar como tratamiento inicial de los abscesos del músculo psoasilíaco antes de la cirugía.

2. El drenaje quirúrgico expuesto por Cantasdemir, Kara, \& Ceibi, (2003), "está indicado en algunas situaciones bien especificadas". Entre las que mencionan:

- Cuando el drenaje percutáneo guiado por tomografía computarizada no tuvo éxito.

- Si el paciente presenta contraindicaciones para el drenaje percutáneo (trastornos de la coagulación, tratamiento anticoagulante crónico).

- Si el paciente tiene una patología intraabdominal diferente que requiera cirugía (absceso del psoasilíaco por enfermedad de Crohn, el intestino afectado también se puede extirpar en la misma intervención).

\section{Conclusiones}

Durante el desarrollo de la literatura se observó, que los abscesos son una patología con un diagnostico complejo y de difícil manifestaciones clínicas que generalmente, es un epifenómeno o una complicación de otra patología (renal, espinal, sigmoidea) que confunde al especialista llamado inicialmente para manejar el caso. Tener una imagen clínica con un diagnóstico diferencial difícil en el contexto en el que la mayoría de los pacientes tienen enfermedades asociadas, puede llevar a un diagnóstico presuntivo en otra dirección. En tal sentido, es recomendado realizar el diagnóstico diferencial cuando se está en presencia de tumores retroperitoneales, hernia femoral, ganglios inguinales agrandados y aneurisma de la arteria ilíaca.

También se observó, en algunos estudios de caso que la mayoría de los pacientes son hospitalizados inicialmente en otros departamentos (nefrología, neurocirugía, medicina interna) y son trasladados a los servicio de gestión médica a los pocos días 
del ingreso. Lo que ocasiona retrasos en el tratado terapéutico con consecuencias adversas sobre la evolución del paciente. Es imprescindible un abordaje multidisciplinar para poder realizar el diagnóstico correcto en el menor tiempo posible.

Es por ello que el manejo debe optimizarse mediante el uso de estudios de imagen por TC o IRM, que, en la mayoría de los casos, es decisivo para establecer el abordaje terapéutico necesario. El drenaje guiado de las colecciones de psoas, a pesar de haberse nombrado durante la investigación, rara vez es posible debido a la baja accesibilidad del procedimiento, o al imperativo de la urgencia de estos casos.

La evolución de los pacientes estará siempre marcada al tiempo en que se determine la existencia de dicha patología, si bien es cierto, el tratamiento que se emplea es el uso de antibióticos, no es luego de la evaluación y el diagnóstico definitivo cuando se logra recetar un tratamiento adecuado y preciso para dicho absceso. Finalmente se puede concluir, que la literatura y los estudios acerca de este tema son escasos, con baja incidencia y sin acuerdo sobre el correcto algoritmo de diagnóstico y tratamiento a seguir, por lo que se recomienda que los estudios futuros pueden ofrecer puntuaciones de diagnóstico para facilitar la detección rápida de esta patología.

\section{Bibliografía}

Abada, A., Solanoa, D., \& Ibarmiaa, J. (2000). Absceso de psoas: difícil orientación clínica inicial. Enfermedades Infecciosas y Microbiología Clínica, 18(5), 244-245. Recuperado el 19 de Jul de 2021, de https://www.elsevier.es/es-revista-enfermedades-infecciosas-microbiologia-clinica-28-articulo-absceso-psoas-dificil-orientacion-clinica-10710

Bolívar, J. (2015). Investigación Documental. México. Pax.

Cantasdemir, M., Kara, B., \& Ceibi, D. (2003). Computed Tomography-guided Percutaneous Catheter Drainage of Primary and Secondary Iliopsoas Abscesses. Clinical Radiology, 58(10), 811.
doi:10.1016/S0009-9260(03)00274-5

Castro, J. (2016). Técnicas Documentales. México. Limusa.

Davila, A. (2015). Diccionario de Términos Científicos. . Caracas: Editorial Oasis.

Demuren, O., \& Abomelha, M. (2002). Iliopsoas abscesses. Saudi Med., 23, 96. doi:doi: 10.1136/ pgmj.2003.017665

Ion, D., Socea, B., Bolocani, A., Paduraru, D., \& Andronic, O. (2020). Psoas Muscle Abscess. REVISTA.CHIMIE, 71(1), 1-6. Recuperado el 22 de Jul de 2021, de https://www.revistadechimie.ro/ pdf/46\%20ION\%20DANIEL\%201\%2020.pdf

Mallick, I., Thoufeeq, M., \& Rajendran, T. (10 de Feb de 2004). Iliopsoas abscesses. Postgraduate Medical Journal, 80, 459-462. doi:http://dx.doi. org/10.1136/pgmj.2003.017665

Ruiz, V., Candel, M., Ruiz, M., \& Peña, E. (2014). Absceso de psoas como diagnóstico diferencial en la patología abdominal de Urgencias. Cirugía General y del Aparato Digestivo, 82, 268-273. Recuperado el 20 de Jul de 2021, de https://www. medigraphic.com/pdfs/circir/cc-2014/cc143e.pdf

Shieldsa, P., Robinsonb, T., \& Crowley, D. (2012). Iliopsoas abscess - A review and update on the literature. International Journal of Surgery, 10(9), 466469. doi:https://doi.org/10.1016/j.ijsu.2012.08.016

Thongngarm, T., \& Mcmurray, R. (2001 ). Primary psoas abscess. Ann Rheum, 60, 173-176. Obtenido de http://ard.bmj.com/

Van Den Berge, M., \& Kuipers, T. (2005). Iliopsoas abscess . Neth. J. Med., 63(10), 413. Recuperado el 20 de Jul de 2021

Wong, O., \& Lam, S. (22 de Abr de 2013). Retrospective review of clinical presentations, microbiology, and outcomes of patients with psoas abscess. Hong Kong Med, 19(5), 416-23. doi:10.12809/ hkmj133793 


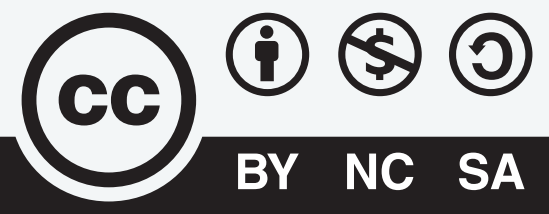

CREATIVE COMMONS RECONOCIMIENTO-NOCOMERCIAL-COMPARTIRIGUAL 4.0.

\section{CITAR ESTE ARTICULO:}

Ronquillo Saavedra, A. A., Rodríguez Cabrera, D. N., \& Guerrero Cedeño, C. B. (2021). Causas de absceso del musculo psoas. RECIMUNDO, 5(3), 188-196. https://doi.org/10.26820/recimundo/5.(2).julio.2021.188-196 